\title{
Estimating treatment importance in multidrug-resistant tuberculosis using Targeted Learning: an observational individual patient data network meta-analysis
}

\author{
Guanbo Wang ${ }^{1}$, Mireille E. Schnitzer ${ }^{2,3, *}$, Dick Menzies ${ }^{4,5}$, Piret Viiklepp ${ }^{6}$, \\ Timothy H. Holtz ${ }^{7}$, and Andrea Benedetti ${ }^{1,4,5}$ \\ ${ }^{1}$ Department of Epidemiology, Biostatistics and Occupational Health, \\ McGill University, Montréal, Québec H3A1A2, Canada \\ ${ }^{2}$ Faculty of Pharmacy, Université de Montréal, Montréal, Québec H3C3J7, Canada \\ ${ }^{3}$ Department of Social and Preventive Medicine, \\ Université de Montréal, Montréal, Québec H3N1X9, Canada \\ ${ }^{4}$ Respiratory Epidemiology and Clinical Research Unit, \\ McGill University Health Centre, Montréal, Québec H4A3S5, Canada \\ ${ }^{5}$ Department of Medicine, McGill University, Montréal, Québec H3H2R9, Canada \\ ${ }^{6}$ National Institute for Health Development, Hiiu 42, Tallinn 11619, Estonia \\ ${ }^{7}$ Division of Global HIV and TB, Centers for Disease Control and Prevention, Atlanta, GA, USA. \\ *email: mireille.schnitzer@umontreal.ca \\ Summary: Persons with multidrug-resistant tuberculosis (MDR-TB) have a disease resulting from a strain of \\ tuberculosis (TB) that does not respond to at least isoniazid and rifampicin, the two most effective anti-TB drugs. \\ MDR-TB is always treated with multiple antimicrobial agents. Our data consist of individual patient data from \\ 31 international observational studies with varying prescription practices, access to medications, and distributions \\ of antibiotic resistance. In this study, we develop identifiability criteria for the estimation of a global treatment \\ importance metric in the context where not all medications are observed in all studies. With stronger causal \\ assumptions, this treatment importance metric can be interpreted as the effect of adding a medication to the existing \\ treatments. We then use this metric to rank 15 observed antimicrobial agents in terms of their estimated add-on value. \\ Using the concept of transportability, we propose an implementation of targeted maximum likelihood estimation \\ (TMLE), a doubly robust and locally efficient plug-in estimator, to estimate the treatment importance metric. A \\ clustered sandwich estimator is adopted to compute variance estimates and produce confidence intervals. Simulation
}




\section{$\operatorname{xxxxxx} \operatorname{xxxx}$}

studies are conducted to assess the performance of our estimator, verify the double robustness property, and assess the appropriateness of the variance estimation approach.

KEY WORDS: Double robustness; Individual patient data; Meta-analysis; Multidrug-resistant tuberculosis; Targeted maximum likelihood estimation; Transportability; Treatment importance. 


\section{Introduction}

Tuberculosis (TB) is an infectious disease caused by bacteria belonging to Mycobacterium tuberculosis complex that predominantly attack the lungs. Multidrug-resistant tuberculosis (MDR-TB) is a type of TB that is resistant to at least isoniazid and rifampin, the two most commonly prescribed TB drugs (Nathanson et al., 2010). MDR-TB is more challenging to treat than drug sensitive TB, requiring a combination of multiple antimicrobial agents, many of which cause serious side effects. The World Health Organization (2018) estimated that 490,000 people worldwide developed MDR-TB in 2016. It was also predicted that the percentage of MDR-TB among incident cases of tuberculosis will increase (Sharma et al., 2017). Therefore, identification of the most effective drug combinations for MDR-TB is urgently needed.

In this setting, statistical analysis of observational data is complicated because patients with MDR-TB usually take multiple treatments and each patient's MDR-TB infection may be resistant to various drugs. Previous work investigating treatment effectiveness in MDR-TB (e.g. Sotgiu et al., 2012; Koh et al., 2013) were small-sample observational studies contrasting a small number of antimicrobial agents.

Meta-analysis is often employed to obtain a global conclusion from numerous studies. In particular, network meta-analysis refers to the synthesis of studies making comparisons between multiple treatments (Mills et al., 2012). When combining multiple studies' individual patient data (IPD), one can obtain additional power and external validity. A comprehensive study by Ahuja et al. (2012) compiled the IPD from 31 observational studies of the relative effectiveness of antimicrobial agents in MDR-TB. Their meta-analysis suggested that the use of later generation quinolones, ofloxacin, and ethionamide/prothionamide as part of multi-drug regimens were associated with treatment success from MDR-TB. They used a random effects model conditional on potential confounders to estimate the adjusted odds ratios and $95 \%$ 
confidence intervals of treatment success associated with different treatments (Ahuja et al., 2012). However, the parametric models used assumed the absence of treatment interactions, and did not take into consideration possible selection bias arising from the usage of only the subset of studies where the treatment was present to evaluate each antimicrobial agent. We are interested in developing methods to investigate associations between antimicrobial agent usage and treatment success while relaxing the implicit parametric and random treatment availability assumptions of the previous analyses.

In this paper, we apply Targeted Maximum Likelihood Estimation (TMLE), a class of methods proposed by van der Laan and Rubin (2006). TMLE, like its semiparametric contemporaries (Robins et al., 1994; Robins, 2000), allows for the relaxation of the strong assumptions made by parametric models. Flexible prediction methods, in particular the ensemble learner called "Super Learner" (van der Laan et al., 2007), are often recommended for the estimation of nuisance components (such as the propensity score and conditional expectation of the outcome) in TMLE (van der Laan and Rose, 2011). If these components are consistently estimated - at a fast-enough rate and with some regularity conditions then for a given, typically nonparametric, model space (van der Laan and Rose, 2011, Section 1.3.1) the TMLE can be constructed to have the lowest asymptotic variance amongst regular asymptotically linear semiparametric estimators (van der Laan and Rubin, 2006). In addition, TMLE for the estimation of causal or censored data parameters can be made doubly robust, meaning that if either of two nuisance components is consistently estimated, the estimator of the parameter of interest is consistent (van der Laan and Rose, 2011).

In this analysis, we use the IPD from Ahuja et al. (2012) consisting of over 9000 patients from 31 studies. In this data, not all antimicrobial agents were used in every study. Therefore data pooling to estimate a parameter defined on a general population requires assumptions relating the available data (where model fitting is carried out) to the target population 
(where inference is desired) (Bareinboim and Pearl, 2016). How to conduct transportability analysis from multiple populations has been discussed by Pearl et al. (2014); Bareinboim and Pearl (2014). Rudolph and van der Laan (2017) developed a TMLE for transporting intervention effects from a fully observed population to a target population.

We aim to estimate a treatment importance metric (van der Laan, 2006) defined as the difference in adjusted probability of treatment success between the patients who used the treatment and the entire population. We develop identifiability (transport) criteria and apply TMLE methods in the setting where not all treatments are available in every study. We conduct a simulation study to test our approach. Finally, we estimate the treatment importance for each of the 15 observed antimicrobial agents used to treat MDR-TB, adjusting for confounding and concurrent treatments. With stronger causal assumptions, the treatment importance could be interpreted as the effect of adding a medication to the existing treatments.

\section{Fused Observational Studies of Patients with MDR-TB}

The application data consist of IPD from international observational studies evaluating treatment effectiveness in MDR-TB, as identified in a systematic review (Ahuja et al., 2012). In particular, studies were identified from three previous systematic reviews (Akçakır, 2009; Orenstein et al., 2009; Johnston et al., 2009) and requests for the IPD were sent to the authors of each study. Web Figure 1 contains details of the data inclusion and exclusion.

\subsection{Data Structure}

The combined dataset contains the IPD from 31 observational studies and a total of 9290 patients. We define the binary outcome $Y$ as treatment success: cure vs. treatment failure, relapse, or death (Falzon et al., 2011). A patient's realization is defined using lowercase $y_{i j}$ where the index $(i, j)$ represents a patient $i \in \mathcal{S}_{j}$ in study $j \in(1, \ldots, 31)$ where $\mathcal{S}_{j}$ is the set of 
indices of patients in study $j$. We also define 15 indicator variables $A^{(k)}$ representing the use of each antimicrobial agent, where $k \in(1, \ldots, 15)$ indexes the antimicrobial agent. Patients may take multiple agents concurrently. We consider 2 study-level covariates: the start year of MDR-TB treatment and the income group of countries of the study, and jointly denote them as $\mathbf{V}$. There are 6 individual-level covariates, namely age, sex, HIV status, acid fast bacilli (AFB) smear status at start of MDR-TB treatment, past history of TB, and cavitation status on X-ray, jointly denoted W. Resistance status $R^{(k)}$ denotes whether a patient had an infection that was tested and determined to be resistant to medication $k$. We define treatment availability (or treatment access) $D^{(k)}$ as follows: we say that the treatment $k$ is available to individual $i$ in study $j$ if at least one of the patients in study $j$ used the treatment $k$ so that $d_{i j}^{(k)}=1$, and otherwise $d_{i j}^{(k)}=0$. Given the presence of missing data in the outcome $Y$, we define an indicator $C$ such that if the outcome is missing, we assign $c_{i j}=1$, and otherwise 0 . Thus, we define our data structure as $\mathbf{O}=\left[Y(1-C), \mathbf{V}, \mathbf{W}, C,\left\{A^{(k)}, R^{(k)}, D^{(k)} ; k=1, \ldots, 15\right\}\right]$. Note that $Y$ is only observed when $C=0$.

For ease of notation, we define the adjustment set to be used for the estimation of the variable importance of treatment $k$ as $\mathbf{X}^{(k)}=\left[\mathbf{V}, \mathbf{W},\left\{A^{\left(k^{*}\right)} ; \forall k^{*} \in(1, \ldots, 15)\right.\right.$ s.t. $\left.\left.k^{*} \neq k\right\}\right]$. In particular, because patients may take multiple treatments, we include all other treatments as "confounders" when evaluating the treatment importance of antimicrobial agent $k$. We also adjust for study-level covariates that may confound comparisons (Schnitzer et al., 2016). As noted above, different medications were available across studies. In order to define variable importance on the global population we develop potential outcome notation under the counterfactual availability of a given treatment. We respectively define an individual's counterfactual outcome and exposure to treatment as $Y\left\{d^{(k)}=1\right\}$ and $\left.A^{(k)}\left\{d^{(k)}=1\right)\right\}$ as the outcome and treatment that would have been observed had the patient had access to 
treatment $k$ and if the outcome had not been censored (i.e. setting $C=0$ ). We provide more intuition for this notation in Web Appendix A.

\subsection{Parameter of Interest \&3 Assumptions}

As in Schnitzer et al. (2016), we consider a hypothetical infinite number of independent studies from which we assume our sample of studies is randomly drawn. Each study then samples patients independently from a possibly distinct study-specific super population. Our target population of interest is the "meta" (or global) population, the combination of all study-specific super populations. We allow for heterogeneity of the treatment effects and assignments, and clustering of covariates and outcomes by study due to measured and unmeasured characteristics of the study-specific populations. The parameters of interest are defined at the individual level over the meta population. We provide more details in Web Appendix B, where we define a non-parametric structural equation model (NPSEM) that encodes the assumed time-ordered data generating structure. In equation (3) of the Web Appendix we derive the counterfactual probability density function (pdf) under the NPSEM.

We are interested in estimating a treatment importance metric for each of the 15 antimicrobial agents observed in the pooled studies. We define these treatment-specific parameters in the subpopulations of patients whose infections were not known to be resistant to the given treatment in order to evaluate treatments in the subpopulations where they may be effective. Under the counterfactual pdf, the treatment importance $\psi^{(k)}$ is defined as

$$
\begin{aligned}
\psi^{(k)} & =E\left(E\left[Y\left\{d^{(k)}=1\right\} \mid \mathbf{X}^{(k)}, R^{(k)}=0, A^{(k)}\left\{d^{(k)}=1\right\}=1\right] \mid R^{(k)}=0\right)-E\left\{Y \mid R^{(k)}=0\right\} \\
& =\tau^{(k)}-\mu^{(k)}
\end{aligned}
$$

This is a contrast between $\tau^{(k)}$, the adjusted probability of treatment success of patients taking treatment $k$ had all patients had access to the treatment $k$, and $\mu^{(k)}$, the probability of treatment success, among the patients whose infections were not known to be resistant to treatment $k$. Both parameters also implicitly impose observed outcomes (no censoring), 
though notation indicating this is suppressed. The contrast is expressed on the risk difference scale and reflects the adjusted improvement in outcomes in those who were taking medication $k$ if it were made available relative to the average outcome.

Identifiability of this parameter requires a list of strong assumptions that allow us to write the parameter of interest in terms of the distribution of the observed data. We list the major assumptions (A1 - A3) in this section and briefly discuss their plausibility.

A1 Consistency: (Needed only for $\tau^{(k)}$.) We assume that the counterfactual treatment and outcome of the individual — had the treatment been available to the individual — are the same when we observe that the treatment was in fact available in their study. I.e., $Y\left\{d^{(k)}=1\right\}=Y$ when $D^{(k)}=1$. We assume the same for the counterfactual treatment, i.e., $A^{(k)}\left\{d^{(k)}=1\right\}=A^{(k)}$ when $D^{(k)}=1$. The second consistency assumption may fail if not all patients in the same study had access to the same treatments (due to the studies being conducted across multiple centers or over long periods of time).

A2 Positivity: (a) (Needed only for $\tau^{(k)}$.) There must be a positive probability of receiving treatment $k$, had the treatment $k$ been available in study $j$, over all possible realizations $x^{(k)}$ of $\mathbf{X}^{(k)}$ among patients whose infections are not known to be resistant to the treatment $k$, i.e., $\operatorname{Pr}\left[A^{(k)}\left\{d^{(k)}=1\right\}=1 \mid \mathbf{X}^{(k)}=x^{(k)}, R^{(k)}=0\right]>0$. Given that not all resistance information was captured in all studies, misclassification of resistance may result in unobserved positivity violations where some included patients whose infections were actually resistant were therefore not eligible to be treated by a given medication.

(b) (Needed only for $\tau^{(k)}$.) There is a positive probability for every study (given any possible characteristic $\mathbf{v}$ ) to have had access to treatment $k$, i.e., $\operatorname{Pr}\left\{D^{(k)}=1 \mid \mathbf{V}=\mathbf{v}\right\}>0$. This assumption will be used to balance out the study-level covariates so that we remove the study-level confounding. This assumption is most likely false as, due to the evolution of practice, certain antimicrobial agents are only observed during certain time frames. The 
small number of studies made this assumption difficult to verify empirically. Failures of the positivity assumptions mean that we are relying on an extrapolation of the outcome model to provide estimates of our parameter of interest.

(c) For all possible values of $\mathbf{x}^{(k)}$, there is a positive probability of observing all outcomes among the patients taking treatment $k$ and whose infections are not known to be resistant to treatment $k$. For $\tau^{(k)}$ the assumption is $\operatorname{Pr}\left\{C=0 \mid A^{(k)}=1, \mathbf{X}^{(k)}=\mathbf{x}^{(k)}, R^{(k)}=0\right\}>0$. For $\mu^{(k)}$ the probabilities are instead evaluated at the observed $A^{(k)}=a_{i j}^{(k)}$. This probability would fail if patients with certain covariate strata never had observed outcomes. We did not detect any empirical evidence that this assumption was violated.

A3. Conditional exchangeability (transportability): (a) (Needed only for $\tau^{(k)}$.) The counterfactual outcomes are independent of treatment availability conditional on confounders in the subset of $\left[R^{(k)}=0, A^{(k)}\left\{d^{(k)}=1\right\}=1\right]$, i.e. $Y\left\{d^{(k)}=1\right\} \Perp D^{(k)} \mid \mathbf{X}^{(k)}, R^{(k)}=$ $0, A^{(k)}\left\{d^{(k)}=1\right\}=1$. This assumption may fail if a study-level variable was correlated with the outcome and the treatment availability simultaneously, and was not accounted for. For example, measures of the study populations' access to health care such as the country's income group or per capita health budget should be included in the adjustment set. It similarly fails if the potential outcomes in studies without access to treatment $k$ are not well represented by the observed outcomes of patients in other studies who did have access to treatment $k$, conditional on covariates. While this assumption is tenuous, it is necessary to estimate parameters interpreted on the union of study populations, which is the goal of obtaining a global measure of effect in meta-analysis.

(b) The censoring is independent of the outcome conditional on the confounders and treatment assignment in the subset of patients whose infections were not known to be resistant to treatment $k$, i.e. $C \Perp Y \mid \mathbf{X}^{(k)}, R^{(k)}=0, A^{(k)}$. This assumption corresponds with the ignorability of censoring and would fail if censoring is related to the unobserved outcome 
beyond the observed covariates. Given the small amount of censoring in our study (2.8\%) we are not overly concerned about violations of this assumption.

Under the assumptions A1 - A3 above, we rewrite our parameter of interest (1) as:

$$
\begin{aligned}
\psi^{(k)}= & E\left[E\left\{Y \mid \mathbf{X}^{(k)}, R^{(k)}=0, A^{(k)}=1, C=0\right\} \mid R^{(k)}=0\right] \\
& -E\left[E\left\{Y \mid \mathbf{X}^{(k)}, R^{(k)}=0, A^{(k)}, C=0\right\} \mid R^{(k)}=0\right] \\
= & \tau^{(k)}-\mu^{(k)}
\end{aligned}
$$

A proof is provided in Web Appendix C. Because $\tau^{(k)}$ and $\mu^{(k)}$ are both estimable from the observed data under the pdf in Web Appendix B equation $(2), \psi^{(k)}$ is identifiable.

With different (sometimes stronger) assumptions, our parameter of interest may be interpreted as a causal parameter: the "add-on" effect independently attributed to treatment $k$ in a fully causal framework. More specifically, this add-on effect would correspond to treatment $k$ 's independent contribution to the probability of treatment success in the population of individuals whose MDR-TB is not resistant to treatment $k$, had all these patients used this treatment in addition to all other treatments they were already taking. Due to the connection between treatment importance and the add-on effect, one might hypothesize that if an antimicrobial agent possesses a large treatment importance, it is possibly an effective add-on medication.

\section{Models \& Algorithm}

In this section, we introduce three estimators of $\tau^{(k)}$, the last of which is the TMLE. Almost identical procedures can be applied to estimate $\mu^{(k)}$. The first estimator requires the estimation of a quantity $Q$, the conditional expectation of the outcome. The second requires the estimation of $g$, the propensity score. The TMLE requires the estimation of both of these quantities. 


\subsection{Outcome Model}

For the estimation of $\tau^{(k)}$, we define $Q^{(\tau k)}=\operatorname{Pr}\left[Y\left\{d^{(k)}=1\right\}=1 \mid A^{(k)}\left\{d^{(k)}=1\right\}=\right.$ $\left.1, \mathbf{X}^{(k)}, R^{(k)}=0\right]$, which is the probability of having a successful counterfactual outcome conditional on the counterfactual treatment exposure and covariates among the patients whose infections were not known to be resistant to treatment $k$. Under the assumptions made above, we show that $Q^{(\tau k)}=\operatorname{Pr}\left\{Y=1 \mid \mathbf{X}^{(k)}, R^{(k)}=0, A^{(k)}=1, C=0\right\}$ in the Web Appendix C. This probability may be estimated by fitting a model using only patients whose infections were not known to be resistant to treatment $k$ (the subset where $R^{(k)}=0$ ) and who were taking the treatment $k$ (the subset where $A^{(k)}=1$ ). Since treatment $k$ is not observed in all studies we use a working assumption that the outcome model estimated on the subset of patients with $D^{(k)}=1$ applies to patients in all studies conditional on measured covariates. Even if assumption A3 holds, this modeling assumption may fail if underlying care is substantially different between studies with different medication availabilities because the marginal model conditional only on observables would not be common in this case.

In the application, we use the R SuperLearner package (van der Laan et al., 2007) to fit a model for $Q^{(\tau k)}$ using the data of patients in the subset where $\left\{r_{i j}^{(k)}=0, a_{i j}^{(k)}=1, c_{i j}=0\right\}$. We then predict values of $Q^{(\tau k)}$ for patients in the subset where $\left\{r_{i j}^{(k)}=0\right\}$ using this model fit. In the simulation study, we use logistic regression (corresponding with the simple datagenerating model) instead of Super Learner. The predicted values are denoted as $Q_{i j, n}^{(\tau k)}$, which are the estimates of $Q^{(\tau k)}$ at the observed value $\mathbf{x}_{i j}^{(k)}$.

Finally, we use G-computation (Robins, 1986) to estimate $\tau^{(k)}$.

$$
\tau_{G c o m p, n}^{(k)}=\frac{1}{n^{(k)}} \sum_{j=1}^{31} \sum_{i \in \mathcal{S}_{j}: r_{i j}^{(k)}=0} Q_{i j, n}^{(\tau k)}
$$

where $n^{(k)}$ is the number of patients whose infections were not known to be resistant to treatment $k$, and $\tau_{\text {Gcomp }, n}^{(k)}$ is the G-computation estimation of $\tau^{(k)}$. 


\subsection{Propensity Score Model}

The propensity score, defined below, is used in Inverse Probability Weighting (IPW) and TMLE to estimate $\tau^{(k)}$. Here, we define the propensity score as: $g^{(\tau k)}=\operatorname{Pr}\left\{A^{(k)}=1, C=\right.$ $\left.0 \mid \mathbf{X}^{(k)}, R^{(k)}=0\right\}$, that is, the probability of being treated according to $k$ and having an uncensored outcome. Under the assumptions described, we decompose $g^{(\tau k)}$ into three parts: $g^{(\tau k)}=\underbrace{\operatorname{Pr}\left\{A^{(k)}=1 \mid D^{(k)}=1, \mathbf{X}^{(k)}, R^{(k)}=0\right\}}_{g_{1}^{(\tau k)}} \underbrace{\operatorname{Pr}\left\{D^{(k)}=1 \mid \mathbf{V}\right\}}_{g_{2}^{(\tau k)}} \underbrace{\operatorname{Pr}\left\{C=0 \mid \mathbf{X}^{(k)}, R^{(k)}=0, A^{(k)}=1\right\}}_{g_{3}^{(\tau k)}}$. Details are provided in Web Appendix D. The first and third components represent the conditional probabilities of being treated according to $k$ and being uncensored, respectively. The second component $g_{2}^{(\tau k)}$ represents the probability of the treatment $k$ being available in study $j$ and can be estimated by regression using the studies as the unit of analysis. This estimated probability can then be assigned to all patients in study $j$. The IPW estimate is

$$
\tau_{I P W, n}^{(k)}=\frac{1}{n^{(k)}} \sum_{j=1}^{31} \sum_{i \in \mathcal{S}_{j}: r_{i j=0}^{(k)}} y_{i j} \frac{\mathbb{1}\left\{a_{i j}^{(k)}=1, c_{i j}=0\right\}}{g_{i j, n}^{(\tau k)}}
$$

where $\mathbb{1}\{\cdot\}$ denotes the indicator function and $g_{i j, n}^{(\tau k)}$ is the estimated propensity score. We prove that the IPW estimator is consistent for $\tau^{(k)}$ in Web Appendix E.

\subsection{TMLE Algorithm}

The TMLE of $\tau^{(k)}$ is doubly robust in the sense that it will be consistent when the estimation of either $Q^{(\tau k)}$ or $g^{(\tau k)}$ would produce a consistent G-computation or IPTW estimator, respectively. Thus, it may have lower bias compared to the singly robust approaches in some circumstances. van der Laan and Rubin (2006) and van der Laan and Rose (2011) describe the asymptotic properties of general TMLEs thoroughly. We briefly outline a TMLE algorithm for our setting below, with full details in Web Appendix F.

We first estimate the components $Q^{(\tau k)}$ and $g^{(\tau k)}$. TMLE takes the initial estimate $Q_{i j, n}^{(\tau k)}$ and "updates" it by borrowing information from the estimated propensity score $g_{i j, n}^{(\tau k)}$. We follow the procedure that fits a logistic regression in the subset of patients whose infections were 
not known to be resistant to medication $k$, and who have observed outcomes. This regression has outcome $Y$, no intercept term, offset $\operatorname{logit}\left\{Q^{(\tau k)}\right\}$, and single covariate $A^{(k)} / g^{(\tau k)}$. We take predictions from this regression fit while setting $A^{(k)}=1$ for all patients not known to be resistant, which we define as the updated values $Q_{i j, n}^{(\tau k)^{*}}$. We then take the average over patients such that $\tau_{T M L E, n}^{(k)}=1 / n^{(k)} \sum_{j=1}^{31} \sum_{i \in \mathcal{S}_{j}::_{i j}^{(k)}=0} Q_{i j, n}^{(\tau k)^{*}}$ is the TMLE estimate.

Following similar steps, we can obtain the TMLE estimate for $\mu$, i.e. $\mu_{T M L E, n}^{(k)}$. For this case we use $Q^{(\mu k)}=\operatorname{Pr}\left\{Y=1 \mid \mathbf{X}^{(k)}, A^{(k)}, R^{(k)}=0, C=0\right\}$, and $g^{(\mu k)}=\operatorname{Pr}\{C=0 \mid$ $\left.\mathbf{X}^{(k)}, A^{(k)}, R^{(k)}=0\right\}$. We fit an intercept-free logistic regression with the unresistant patients with observed outcomes, offset $\operatorname{logit}\left\{Q^{(\mu k)}\right\}$ and single covariate $1 / g^{(\mu k)}$. This fit is used to predict outcomes for all unresistant patients and these predictions are averaged over to get $\mu_{T M L E, n}^{(k)}$. Finally, the TMLE estimate of the parameter of interest is: $\psi_{T M L E, n}^{(k)}=$ $\tau_{T M L E, n}^{(k)}-\mu_{T M L E, n}^{(k)}$.

Clustering by study is taken into consideration in the estimation of the variance where studies are assumed to be the independent units. We use the influence curve approach described in Schnitzer et al. (2014), which is only valid when both $Q$ and $g$ components are consistently estimated and when the number of studies (clusters) is sufficiently large. Details of the variance estimation are available in Web Appendix F.2.

\subsection{Simulation Study}

We performed a simulation study where we applied our estimator to simulated IPD demonstrating 1) the consistency and double robustness of the estimator under study heterogeneity (including treatment effects that vary by study), 2) the appropriateness of the variance estimation and the coverage of the Wald-type confidence intervals based on the influence curve approach for different sample sizes, and 3) the potential importance of considering treatment availability (transportability) in our setting. Details and results are available in the Web Appendix G. 


\section{Results of Analysis of MDR-TB Data}

IPD were obtained from multiple observational studies that investigated associations between drugs taken and treatment success in patients with MDR-TB. Study level information including the numbers of patients and treatments used in each cohort is presented in Table 1. Patient-level summaries for each covariate and outcome are given in Web Table 7.

[Table 1 about here.]

The year of study ranged between 1995 and 2009. Of the pooled data, 404 (4.4\%) and $3106(33.4 \%)$ of patients came from low middle income group countries and upper middle income group countries respectively, and the remaining 5780 (62.2\%) patients came from high income group countries. Study-specific sample sizes ranged between 25 and 2211 patients. Some studies only observed 3 drugs used on their population while others observed all 15 drugs. The range of the mean age across studies was 31.1 to 47.6, except for one pediatric study; 26 studies included pediatric patients, totalling 255 overall. The inclusion of patients coinfected with HIV varied substantially between studies as did the distributions of the other covariates. In terms of outcome, $4847(52.2 \%)$ patients had a clinically successful outcome, while no outcome was reported for 260 patients $(2.8 \%)$.

In Table 2 we provide the number of patients prescribed and the number of patients whose infections were resistant to each treatment. Ofloxacin, pyrazinamide, cycloserine, and kanamycin were each prescribed to a majority of patients and a large number of infections were resistant to ethambutol, streptomycin, and pyrazinamide.

[Table 2 about here.]

In the data analysis, we dropped the few observations with missing sex and age information $(0.065 \%$ and $0.301 \%$ respectively). To pragmatically handle missingness in the other binary covariates, we set the missing values to zero, created binary indicators (dummy variables) for missingness, and included these as covariates in the models. Following the procedures 
described in Section 3, the treatment importance of all 15 treatments was estimated with the TMLE algorithm, for which Super Learner was used to estimate the $Q$ component. Super Learner, which uses cross-validation to combine predictions across a library of methods, was implemented with k-nearest neighbors, random forests (Liaw and Wiener, 2002), logistic regression, and LASSO (Friedman et al., 2010). Logistic regressions were used to fit the propensity score models and a LASSO penalty was added when the model otherwise failed to converge. Table 3 shows the estimates, ordered by estimated treatment importance, with their associated clustered influence curve based standard errors and confidence intervals.

[Table 3 about here.]

As shown in the table, ciprofloxacin had the greatest estimated treatment importance of 0.134 (95\%CI: $0.024,0.243)$ meaning that those taking ciprofloxacin had an average probability of treatment success that was 0.134 higher than the general population, after adjusting for confounding and differential treatment access. Other treatments with at least moderately large positive treatment importance were amikacin, High-generation quinolones, capreomycin, ethionamide, streptomycin, and cycloserine. Among these, hypothesis tests for the treatment importance of streptomycin and cycloserine also rejected the null (zero importance). Therefore, with strong assumptions, we may infer that ciprofloxacin would contribute the most to the average improvement in treatment success in the context of multiple treatment usage. There is also evidence that streptomycin and cycloserine both have positive treatment importance. In contrast, para-aminosalicylic acid, pyrazinamide and Group 5 level drugs had negative estimated importance though the confidence intervals included the null. Further, in order to see whether the consideration of transportability made a practical difference, we compared the results of a standard TMLE which ignores treatment availability (Web Table 8) and thus ignores the extrapolation over studies that do not have access to the given treatment. The conclusions changed substantially - in particular, 
we see that ciproflaxin, our top treatment in the primary analysis, no longer appeared to be important. Ethionamide now appears important, as it did in the study by Ahuja et al. (2012) and rifabutin appears harmful (negative treatment importance). The standard errors were almost always smaller when ignoring treatment availability.

Finally, we compared the results of this analysis with a standard logistic regression model with random study-specific intercepts which pooled over all of the data ignoring treatment availability. We chose this comparator as mixed effects regression analysis is a typical approach to one-stage network meta-analysis with IPD (Thomas et al., 2014). In order to fit the model, we removed all observations with missing outcomes. The model included all treatment main terms and adjusted for the same covariates as the primary analysis (as main terms). The estimates are provided in the Web Table 9. The results were generally not consistent with our primary results; streptomycin and ethionamide did not appear as important; prothionamide and ofloxacin were significantly and negatively associated with the outcome, suggesting harm, while kanamycin was significantly and positively associated with the outcome despite sparsity of this treatment. Ciprofloxacin was positively associated with the outcome in both analyses.

\section{Discussion}

Our study derived sufficient assumptions and TMLE methodology for the consistent estimation of a treatment importance parameter under a nonparametric model in the IPD meta-analytical context. In particular, this formalized the extrapolation assumptions that may be necessary to aggregate results over studies when there are many treatment options.

Our perspective and analysis deviated from a traditional meta-analysis. One-stage network meta-analysis typically applies parametric outcome modeling where the patient is the unit of analysis in order to synthesize multiple studies that are each contrasting different sets of treatments (Mills et al., 2012). Two-stage approaches first summarize each study's treatment 
contrasts and then employ an aggregate meta-analysis technique to estimate population average treatment effects. These approaches typically allow for treatment effect heterogeneity across study populations (Thomas et al., 2014) but are limited in their reliance on parametric modeling and do not explicitly consider the estimation of causal quantities nor of transportability (Schnitzer et al., 2016). In our application we found that a simpler mixed model analysis changed the study conclusions substantially. Our one-stage approach explicitly allows for the common setting where treatments are informatively distributed across studies and allows for between-study heterogeneity of the treatment effects. Additional strengths of our methods include the double robustness of TMLE and the handling of missing-at-random outcomes. Our methods can be implemented non-parametrically (e.g. with Super Learner) which may avoid model misspecification. A limitation of our approach is that it is difficult to implement for very small numbers of studies. In particular, we take study as the unit of measurement in the second propensity score model which limits the ability to adjust for study-specific covariates. In this study we used a LASSO penalty when the model for $g_{2}^{(\tau k)}$ did not converge. Previous work used standard outcome regression methods to estimate adjusted odds ratios of MDR-TB treatment success separately for each medication (Ahuja et al., 2012). Our analysis focused on the nonparametrically defined treatment importance of each antimicrobial agent, allowing for the identification of medications associated with treatment success. We did not focus on a parameter involving an intervention on treatment usage a priori because we felt that the required causal identifiability assumptions would not be not credible in our setting. Rather than use a standard risk difference which contrasts outcomes in those taking versus not taking a medication, we chose to contrast the adjusted probability of treatment success in those taking each medication with the probability under observed usage. Our chosen parameter approximates the expected improvement in outcomes if a medication were to be added on to all regimens where it was not already included. The alternative contrast 
would instead approximate the comparison of outcomes under adding on versus potentially removing a medication, which we found to be less clinically relevant. It may also be of interest to directly compare the importance of two different medications. Because we evaluated each treatment in the subpopulation with infections not known to be resistant to the antimicrobial agent (Lew et al., 2008), the target populations are not common for each medication. For direct comparisons between medications, one may consider a variable importance parameter evaluated on the relative risk scale. Other studies, including our past work, reported the success of regimens/combinations of treatments, rather than individual treatments (Siddique et al., 2018; Masjedi et al., 2008; Kuaban et al., 2015).

While the goal of our analysis was to estimate a global treatment importance parameter, substantial heterogeneity in treatment effects across studies may make this parameter less clinically relevant. Future work in our group will involve investigation of treatment effect modification and optimal regimens based on clinical baseline covariates.

In practice, investigators may be inclined to conduct their systematic reviews based on studies that evaluated a specific treatment of interest. In the simulation study, we showed that ignoring studies with only alternative treatments for the same condition may lead to bias. An important conclusion is that, when estimating population average effects and parameters, analyses should not be restricted to only data from studies that included a given treatment without adjustment for selection bias.

\section{Acknowledgments}

This work was supported by the Canadian Institutes of Health Research [Project Grant to MES and AB, and a New Investigators Salary Award to MES. The data and context were provided by the Collaborative Group for Meta-Analysis of Individual Patient Data in Multidrug-Resistant Tuberculosis. We thank Dr. Tjip S van der Werf for his comments on the manuscript. Conflict of Interest: None declared. 


\section{References}

Ahuja, S. D., Ashkin, D., Avendano, M., Banerjee, R., Bauer, M., Bayona, J. N., Becerra, M. C., Benedetti, A., Burgos, M., Centis, R., et al. (2012). Multidrug resistant pulmonary tuberculosis treatment regimens and patient outcomes: an individual patient data metaanalysis of 9,153 patients. PLOS Medicine $\mathbf{9}$, e1001300.

Akçakır, Y. (2009). Correlates of treatment outcomes of multidrug-resistant tuberculosis (MDR-TB): a systematic review and meta-analysis. Master's thesis, McGill University.

Bareinboim, E. and Pearl, J. (2014). Transportability from multiple environments with limited experiments: Completeness results. pages 280-288. Advances in Neural Information Processing Systems 27.

Bareinboim, E. and Pearl, J. (2016). Causal inference and the data-fusion problem. PNAS 113, $7345-7352$.

Falzon, D., Jaramillo, E., Schünemann, H. J., Arentz, M., Bauer, M., Bayona, J., Blanc, L., Caminero, J. A., Daley, C. L., Duncombe, C., et al. (2011). WHO guidelines for the programmatic management of drug-resistant tuberculosis: 2011 update.

Friedman, J., Hastie, T., and Tibshirani, R. (2010). Regularization paths for generalized linear models via coordinate descent. Journal of Statistical Software 33, 1-22.

Johnston, J. C., Shahidi, N. C., Mohsen, S. S., and Fitzgerald, J. M. (2009). Treatment outcomes of multidrug-resistant tuberculosis: a systematic review and meta-analysis. PLOS ONE 4, e6914.

Koh, W. J., Lee, S. H., Kang, Y. A., Lee, C. H., Choi, J. C., Lee, J. H., Jang, S. H., Yoo, K. H., Jung, K. H., Kim, K. U., et al. (2013). Comparison of levofloxacin versus moxifloxacin for multidrug-resistant tuberculosis. American Journal of Respiratory and Critical Care Medicine 188, 858-864.

Kuaban, C., Noeske, J., Rieder, H. L., Ait-Khaled, N., Foe, J. L. A., and Trébucq, A. 
(2015). High effectiveness of a 12-month regimen for MDR-TB patients in cameroon. The International Journal of Tuberculosis and Lung Disease 19, 517-524.

Lew, W., Pai, M., Oxlade, O., Martin, D., and Menzies, D. (2008). Initial drug resistance and tuberculosis treatment outcomes: systematic review and meta-analysis. Annals of Internal Medicine 149, 123-134.

Liaw, A. and Wiener, M. (2002). Classification and regression by randomforest. $R$ News $\mathbf{2}$, $18-22$.

Masjedi, M. R., Tabarsi, P., Chitsaz, E., Baghaei, P., Mirsaeidi, M., Amiri, M. V., Farnia, P., Javanmard, P., Mansouri, D., and Velayati, A. A. (2008). Outcome of treatment of MDR-TB patients with standardised regimens, iran, 2002-2006. The International Journal of Tuberculosis and Lung Disease 12, 750-755.

Mills, E. J., Ioannidi, J. P., Thorlund, K., Schuenemann, H. J., Puhan, M. A., and Guyatt, G. H. (2012). How to use an article reporting a multiple treatment comparison metaanalysis. The Journal of the American Medical Association 308, 1246-1253.

Nathanson, E., Nunn, P., Uplekar, M., Floyd, K., Jaramillo, E., Lönnroth, K., Weil, D., and Raviglione, M. (2010). MDR tuberculosis - critical steps for prevention and control. New England Journal of Medicine 363, 1050-1058.

Orenstein, E. W., Basu, S., Shah, N. S., Andrews, J. R., Friedland, G. H., Moll, A. P., Gandhi, N. R., and Galvani, A. P. (2009). Treatment outcomes among patients with multidrugresistant tuberculosis: systematic review and meta-analysis. The Lancet Infectious Diseases 9, 153-161.

Pearl, J., Bareinboim, E., et al. (2014). External validity: From do-calculus to transportability across populations. Statistical Science 29, 579-595.

Robins, J. (1986). A new approach to causal inference in mortality studies with a sustained exposure period - application to control of the healthy worker survivor effect. 
Mathematical Modelling 7, 1393-1512.

Robins, J. M. (2000). Robust estimation in sequentially ignorable missing data and causal inference models. In ASA Proceedings of the Section on Bayesian Statistical Science, pages $6-10$.

Robins, J. M., Rotnitzky, A., and Zhao, L. P. (1994). Estimation of regression coefficients when some regressors are not always observed. Journal of the American Statistical Association 89, 846-866.

Rudolph, K. E. and van der Laan, M. J. (2017). Robust estimation of encouragement design intervention effects transported across sites. Journal of the Royal Statistical Society: Series B (Statistical Methodology) 79, 1509-1525.

Schnitzer, M. E., Steele, R. J., Bally, M., and Shrier, I. (2016). A causal inference approach to network meta-analysis. Journal of Causal Inference $\mathbf{4}$.

Schnitzer, M. E., van der Laan, M. J., Moodie, E. M., and Platt, R. W. (2014). Effect of breastfeeding on gastrointestinal infection in infants: a targeted maximum likelihood approach for clustered longitudinal data. The Annals of Applied Statistics 8, 703.

Sharma, A., Hill, A., Kurbatova, E., van der Walt, M., Kvasnovsky, C., Tupasi, T. E., Caoili, J. C., Gler, M. T., Volchenkov, G., Kazennyy, B., et al. (2017). Estimating the future burden of multidrug-resistant and extensively drug-resistant tuberculosis in india, the philippines, russia, and south africa: a mathematical modelling study. The Lancet Infectious Diseases 17, 707-715.

Siddique, A. A., Schnitzer, M. E., Bahamyirou, A., Wang, G., Holtz, T. H., Migliori, G. B., Sotgiu, G., Gandhi, N. R., Vargas, M. H., Menzies, D., and Benedetti, A. (2018). Causal inference with multiple concurrent medications: A comparison of methods and an application in multidrug-resistant tuberculosis. Statistical Methods in Medical Research 28, 3534-3549. 
Sotgiu, G., Centis, R., D’Ambrosio, L., Alffenaar, J. W. C., Anger, H. A., Caminero, J. A., Castiglia, P., Lorenzo, S. D., Ferrara, G., Koh, W. J., et al. (2012). Efficacy, safety and tolerability of linezolid containing regimens in treating MDR-TB and XDR-TB: systematic review and meta-analysis. European Respiratory Journal 40, 1430-1442.

Thomas, D., Radji, S., and Benedetti, A. (2014). Systematic review of methods for individual patient data meta-analysis with binary outcomes. BMC Medical Research Methodology 14, Article 79.

van der Laan, M. J. (2006). Statistical inference for variable importance. The International Journal of Biostatistics 2, Article 2.

van der Laan, M. J., Polley, E. C., and Hubbard, A. E. (2007). Super learner. Statistical Applications in Genetics and Molecular Biology 6, Article 25.

van der Laan, M. J. and Rose, S. (2011). Targeted Learning: Causal Inference for Observational and Experimental Data. Springer Science \& Business Media.

van der Laan, M. J. and Rubin, D. (2006). Targeted maximum likelihood learning. The International Journal of Biostatistics 2, Article 11.

World Health Organization (2018). What is multidrug-resistant tuberculosis (MDR-TB) and how do we control it? http://www.who.int/features/qa/79/en/. Accessed: 2018-0930.

\section{Supporting Information}

Web Appendices, Figures and Tables referenced in Sections $2-4$ are available with this article at the Biometrics website on Wiley Online Library. Code and data from the simulation study are available at https://doi.org/10.5281/zenodo.1405199.

Received month year. Revised month year. Accepted month year. 
Table 1: Study Information. ${ }^{a}$ IG: Income group of the country of each study. ${ }^{b}$ N.: The number of patients in each study. ${ }^{c}$ We use letters to present the treatments. a: Ethambutol; b:Amikacin; c:Capreomycin; d:Ciprofloxacin; e:Cycloserine; f:Ethionamide/Prothionamide; g:Ofloxacin; h:Para-Aminosalicylic Acid; i:Protionamide; j:Rifabutin; k:Streptomycin; l:Pyrazinamide; m:Kanamycin n:High- generation quinolones; o:Group 5 level drugs respectively. ${ }^{d}$ Male N(\%): Number and percentage of males in each study. ${ }^{e}$ HIV N(\%): Number and percentage of HIV positive patients in each study. ${ }^{f}$ Smear N(\%): Number and percentage of patients with positive Acid fast bacilli smear in each study. ${ }^{g}$ TB N(\%): Number and percentage of patients with past positive TB strains in each study. ${ }^{h}$ Cav. $\mathrm{N}(\%)$ : Number and percentage of patients with positive cavitation on X Ray in each study.

\begin{tabular}{|c|c|c|c|c|c|c|c|c|c|c|}
\hline Author name & Year & $\mathbf{I G}^{a}$ & $\mathbf{N}^{b}$ & Trts. used ${ }^{c}$ & Age mean (SD) & Male $\mathbf{N}(\%)^{d}$ & HIV $\mathbf{N}(\%)^{e}$ & Smear $\mathbf{N}(\%)^{f}$ & TB N $(\%)^{g}$ & Cav. $\mathbf{N}(\%)^{h}$ \\
\hline Ahuja & 1997 & High & 823 & $a-h, j-o$ & $41.4(12.0)$ & $561(68.2)$ & $488(59.3)$ & $509(61.8)$ & $0(0.0)$ & $0(0.0)$ \\
\hline Avendano & 2009 & High & 72 & $a-h, j-o$ & $36.3(15.3)$ & $43(59.7)$ & $1(1.4)$ & $67(93.1)$ & $39(54.2)$ & $0(0.0)$ \\
\hline Burgos & 2000 & High & 48 & $a-h, j-o$ & $47.2(14.8)$ & $32(66.7)$ & $11(22.9)$ & $36(75.0)$ & $31(64.6)$ & $21(43.8)$ \\
\hline Chan & 1998 & High & 203 & $a, c-h, j-o$ & $42.0(14.3)$ & $116(57.1)$ & $0(0.0)$ & $203(100.0)$ & $196(64.4)$ & $120(59.1)$ \\
\hline Chiang & 1996 & High & 125 & $\mathrm{a}, \mathrm{e}, \mathrm{g}-\mathrm{m}, \mathrm{o}$ & $46.1(15.2)$ & $90(72.0)$ & $0(0.0)$ & $109(87.2)$ & $114(91.2)$ & $0(0.0)$ \\
\hline Cox & 2005 & Lower Middle & 77 & $\mathrm{a}, \mathrm{c}, \mathrm{e}-\mathrm{h}, \mathrm{j}-\mathrm{m}, 0$ & $36.9(11.2)$ & $47(61.0)$ & $0(0.0)$ & $76(98.7)$ & $77(100.0)$ & $0(0.0)$ \\
\hline Garcia & 2009 & Upper Middle & 47 & $a, j-o$ & $47.6(16.4)$ & $26(55.3)$ & $0(0.0)$ & $42(89.4)$ & $14(29.8)$ & $20(42.6)$ \\
\hline Granich & 2006 & High & 104 & $a-h, j-m$ & $40.3(19.5)$ & $61(58.7)$ & $1(1.0)$ & $75(72.1)$ & $62(59.6)$ & $0(0.0)$ \\
\hline Koh & 2005 & High & 155 & a,c-e,g-i,k-o & $40.9(14.4)$ & $82(52.9)$ & $0(0.0)$ & $131(84.5)$ & $137(88.4)$ & $0(0.0)$ \\
\hline Leung & 1997 & High & 99 & $\mathrm{e}, \mathrm{g}, \mathrm{l}-\mathrm{m}$ & $46.1(16.2)$ & $74(74.7)$ & $0(0.0)$ & $78(78.8)$ & $62(62.6)$ & $46(46.5)$ \\
\hline Migliori & 2004 & High & 101 & $\mathrm{a}-\mathrm{i}, \mathrm{k}-\mathrm{o}$ & $39.4(14.7)$ & $61(60.4)$ & $6(5.9)$ & $80(79.2)$ & $58(57.4)$ & $48(47.5)$ \\
\hline Mitnick & 2002 & Upper Middle & 732 & $\mathrm{a}-\mathrm{O}$ & $31.1(12.0)$ & $436(59.6)$ & $8(1.1)$ & $508(69.4)$ & $720(98.4)$ & $550(75.1)$ \\
\hline Narita & 1997 & High & 81 & $a-h, j-m, o$ & $40.2(11.8)$ & $55(67.9)$ & $41(50.6)$ & $0(0.0)$ & $53(65.4)$ & $33(40.7)$ \\
\hline Palmero & 1999 & High & 114 & $\mathrm{a}, \mathrm{c}-\mathrm{i}, \mathrm{k}-\mathrm{m}, \mathrm{o}$ & $35.3(13.3)$ & $54(47.4)$ & $0(0.0)$ & $108(94.7)$ & $76(66.7)$ & $103(90.4)$ \\
\hline Pasvol & 2004 & High & 45 & $\mathrm{a}, \mathrm{b}, \mathrm{d}, \mathrm{e}, \mathrm{g}-\mathrm{o}$ & $36.9(15.9)$ & $21(51.2)$ & $0(0.0)$ & $29(70.7)$ & $24(58.5)$ & $0(0.0)$ \\
\hline Pena & 2000 & High & 25 & $\mathrm{a}, \mathrm{h}, \mathrm{o}$ & $41.2(13.3)$ & $24(96.0)$ & $0(0.0)$ & $25(100.0)$ & $22(88.0)$ & $16(64.0)$ \\
\hline Perez & 1995 & Upper Middle & 34 & $\mathrm{a}, \mathrm{b}, \mathrm{d}, \mathrm{g}, \mathrm{i}, \mathrm{k}-\mathrm{m}, \mathrm{o}$ & $42.1(12.4)$ & $21(61.8)$ & $0(0.0)$ & $34(100.0)$ & $34(100.0)$ & $22(64.7)$ \\
\hline Quy & 2000 & Lower Middle & 157 & $a, j-1$ & $39.5(11.4)$ & $121(77.1)$ & $4(2.5)$ & $157(100.0)$ & $102(65.0)$ & $0(0.0)$ \\
\hline Riekstina & 2004 & High & 1027 & a-c,e,g-i,k-o & $42.3(12.7)$ & $780(75.9)$ & $32(3.1)$ & $269(26.2)$ & $648(63.1)$ & $704(68.5)$ \\
\hline Robert & 1999 & High & 45 & a,e-h,j-m,o & $41.7(15.6)$ & $24(53.3)$ & $9(20.0)$ & $33(73.3)$ & $24(53.3)$ & $31(68.9)$ \\
\hline Schaaf & 2002 & Upper Middle & 39 & $a, b, e-g, j-m, o$ & $7.0(5.4)$ & $20(51.3)$ & $6(15.4)$ & $9(23.1)$ & $12(30.8)$ & $14(35.9)$ \\
\hline Seung & 2002 & High & 142 & $\mathrm{a}, \mathrm{e}, \mathrm{g}-\mathrm{i}, \mathrm{k}-\mathrm{m}$ & $43.9(15.4)$ & $117(82.4)$ & $0(0.0)$ & $142(100.0)$ & $142(100.0)$ & $86(60.6)$ \\
\hline Shim & 2002 & High & 1364 & $\mathrm{a}, \mathrm{e}, \mathrm{g}-\mathrm{o}$ & $42.8(14.9)$ & $1014(74.3)$ & $1(0.1)$ & $927(68.0)$ & $977(71.6)$ & $569(41.7)$ \\
\hline Shin & 2004 & High & 608 & a-c,e-i,l-o & $35.8(11.3)$ & $506(83.2)$ & $5(0.8)$ & $497(81.7)$ & $592(97.4)$ & $368(60.5)$ \\
\hline Shiraishi & 2007 & High & 61 & $a, c-f, h, j-o$ & $46.4(11.9)$ & $46(75.4)$ & $0(0.0)$ & $0(0.0)$ & $0(0.0)$ & $61(100.0)$ \\
\hline Tabarsi & 2006 & Upper Middle & 43 & $\mathrm{a}, \mathrm{b}, \mathrm{e}, \mathrm{g}-\mathrm{i}, \mathrm{l}, \mathrm{m}, \mathrm{o}$ & $44.4(19.1)$ & $27(62.8)$ & $0(0.0)$ & $42(97.7)$ & $43(100.0)$ & $43(100.0)$ \\
\hline Tupasi & 2003 & Lower middle & 170 & $\mathrm{a}-\mathrm{O}$ & $39.2(12.4)$ & $106(62.3)$ & $0(0.0)$ & $107(62.9)$ & $164(96.5)$ & $138(181.2)$ \\
\hline Van der Werf & 2008 & High & 43 & $\mathrm{a}, \mathrm{b}, \mathrm{d}-\mathrm{h}, \mathrm{j}, \mathrm{l}-\mathrm{m}, \mathrm{o}$ & $32.9(18.3)$ & $31(73.8)$ & $0(0.0)$ & $0(0.0)$ & $0(0.0)$ & $0(0.0)$ \\
\hline Van der Walt & 2004 & Upper Middle & 2211 & $\mathrm{a}, \mathrm{e}-\mathrm{g}, \mathrm{l}, \mathrm{m}$ & $36.6(10.9)$ & $13965(62.5)$ & $565(25.9)$ & $1376(63.0)$ & $1963(89.9)$ & $1247(57.1)$ \\
\hline Viiklepp & 2002 & High & 284 & $\mathrm{a}-\mathrm{c}, \mathrm{e}, \mathrm{g}-\mathrm{m}, \mathrm{o}$ & $43.0(13.6)$ & $201(70.8)$ & $9(3.2)$ & $153(53.9)$ & $0(0.0)$ & $206(72.5)$ \\
\hline Yim & 2007 & High & 211 & $\mathrm{a}-\mathrm{c}, \mathrm{e}, \mathrm{g}-\mathrm{o}$ & $39.3(15.8)$ & $124(58.8)$ & $0(0.0)$ & $0(0.0)$ & $84(39.8)$ & $160(75.8)$ \\
\hline
\end{tabular}


Table 2: Number using each treatment and with infections resistant to each treatment. * res: patients' infections are resistant to the given treatment. The $\%$ s are taken over the entire sample.

\begin{tabular}{llll}
\hline Treatment & N used (\%) & N res* \& used (\%) & N res \& unused (\%) \\
\hline High-generation quinolones & $930(10.0)$ & & \\
Ciprofloxacin & $1031(11.1)$ & & \\
Prothionamide & $3341(36.1)$ & & \\
Amikacin & $605(6.5)$ & & $180(1.9)$ \\
Cycloserine & $5729(61.9)$ & $299(3.2)$ & $1362(14.7)$ \\
Kanamycin & $5093(55.0)$ & $458(4.9)$ & \\
Ofloxacin & $6538(70.6)$ & & $1806(19.5)$ \\
Group 5 level drugs & $2205(23.8)$ & & $3052(33.0)$ \\
Pyrazinamide & $6263(67.6)$ & $962(10.4)$ & $646(7.0)$ \\
Ethambutol & $4325(46.7)$ & $1075(11.6)$ & $344(3.7)$ \\
Para-Aminosalicylic Acid & $4005(43.3)$ & $446(4.8)$ & $1268(13.7)$ \\
Capreomycin & $1956(21.1)$ & $286(3.1)$ & $3728(40.3)$ \\
Ethionamide & $4005(43.3)$ & $493(5.3)$ & \\
Streptomycin & $1418(15.3)$ & $425(4.6)$ & \\
Rifabutin & $1371(14.8)$ & & \\
\hline
\end{tabular}


Table 3: Treatment importance, associated standard error and confidence interval of 15 treatment.

\begin{tabular}{llll}
\hline Treatment & Estimate & Standard Error & 95\% Confidence Interval \\
\hline Ciprofloxacin & 0.134 & 0.056 & $(0.024,0.243)$ \\
Amikacin & 0.091 & 0.056 & $(-0.018,0.200)$ \\
High-generation quinolones & 0.084 & 0.102 & $(-0.116,0.283)$ \\
Capreomycin & 0.070 & 0.064 & $(-0.055,0.196)$ \\
Ethionamide & 0.068 & 0.040 & $(-0.011,0.147)$ \\
Streptomycin & 0.063 & 0.027 & $(0.011,0.116)$ \\
Cycloserine & 0.054 & 0.028 & $(0.000,0.109)$ \\
Prothionamide & 0.047 & 0.154 & $(-0.255,0.348)$ \\
Ofloxacin & 0.023 & 0.032 & $(-0.040,0.085)$ \\
Ethambutol & 0.020 & 0.022 & $(-0.022,0.063)$ \\
Kanamycin & 0.020 & 0.024 & $(-0.027,0.067)$ \\
Rifabutin & 0.014 & 0.071 & $(-0.125,0.153)$ \\
Para-aminosalicylic acid & -0.002 & 0.019 & $(-0.038,0.035)$ \\
Pyrazinamide & -0.005 & 0.017 & $(-0.038,0.028)$ \\
Group 5 level drugs & -0.035 & 0.037 & $(-0.108,0.038)$ \\
\hline
\end{tabular}

